Draft version August 27, 2018

Preprint typeset using IATEX style AASTeX6 v. 1.0

\title{
THE EXTENDED NORTHERN ROSAT GALAXY CLUSTER SURVEY (NORAS II) I. SURVEY CONSTRUCTION AND FIRST RESULTS
}

\author{
Hans Böhringer ${ }^{1}$, Gayoung Chon $^{1}$, Jörg RetzlafF ${ }^{2}$, Joachim Trümper ${ }^{1}$, Klaus Meisenheimer ${ }^{3}$ And Norbert \\ SCHARTEL $^{4}$
}

\author{
${ }^{1}$ Max-Planck-Institut für extraterrestrische Physik, D-85748 Garching, Germany \\ ${ }^{2}$ ESO, D-85748 Garching, Germany \\ ${ }^{3}$ Max-Planck-Institut für Astronomy, Königstuhl 17, D-69117 Heidelberg, Germany \\ ${ }^{4}$ ESAC, Camino Bajo del Castillo, Villanueva de la Cañada, 28692 Madrid, Spain
}

\section{ABSTRACT}

As the largest, clearly defined building blocks of our Universe, galaxy clusters are interesting astrophysical laboratories and important probes for cosmology. X-ray surveys for galaxy clusters provide one of the best ways to characterise the population of galaxy clusters. We provide a description of the construction of the NORAS II galaxy cluster survey based on X-ray data from the northern part of the ROSAT All-Sky Survey. NORAS II extends the NORAS survey down to a flux limit of $1.8 \times 10^{-12}$

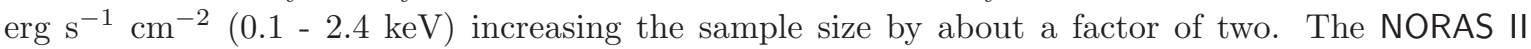
cluster survey now reaches the same quality and depth of its counterpart, the Southern REFLEX II survey, allowing us to combine the two complementary surveys. The paper provides information on the determination of the cluster X-ray parameters, the identification process of the X-ray sources, the statistics of the survey, and the construction of the survey selection function, which we provide in numerical format. Currently NORAS II contains 860 clusters with a median redshift of $z=0.102$. We provide a number of statistical functions including the $\log \mathrm{N}-\log \mathrm{S}$ and the $\mathrm{X}$-ray luminosity function and compare these to the results from the complementary REFLEX II survey. Using the NORAS II sample to constrain the cosmological parameters, $\sigma_{8}$ and $\Omega_{m}$, yields results perfectly consistent with those of REFLEX II. Overall, the results show that the two hemisphere samples, NORAS II and REFLEX II, can be combined without problems to an all-sky sample, just excluding the Zone-of-Avoidance.

Keywords: Surveys, X-rays: galaxies: clusters, Galaxies: clusters: general, Cosmology: observations

\section{INTRODUCTION}

To explore the structure and evolution of our Universe we have to rely on visible objects and observable physical processes. Some of the most interesting systems for such studies are galaxy clusters, the largest properly defined astronomical objects. They allow us to trace the large scale matter distribution (e.g. Peacock \& West 1992, Einsato et al. 1993, Schuecker et al. 2001, Böhringer et al. 2015) and to test cosmological models (e.g. Perenod 1980, Borgani et al. 2001, Henry et al. 2004, 2009, Vikhlinin et al. 2009, Böhringer et al. 2014, Planck Collaboration 2014, 2016). They are also interesting laboratories to study the galaxy population and the physics of the intergalactic medium (e.g. Mulchey, Dressler \& Oemler 2004, Voit 2005, Markevitch \& Vikhlinin 2007, Kravtsov \& Borgani 2012, Eckert et al. 2015, Bykov et al. 2015, Churazov et al. 2016, Arevalo, et al. 2016). Such studies require the systematic construction of statistically complete, well defined samples of galaxy clus- ters.

The detection and characterisation of galaxy clusters by their X-ray emission is still one of the most advanced techniques for building large statistical galaxy cluster samples. Contrary to the optical appearance of clusters, where they have to be characterised as a collection of galaxies, X-rays show clusters as single entities. The X-rays further provide evidence for a deep gravitational potential that binds the matter of the cluster. In addition X-ray surveys yield cluster samples which are approximately mass selected, since the X-ray luminosity is tightly correlated with cluster mass (e.g. Pratt et al. 2009, Böhringer et al. 2012) and the X-ray emission is highly peaked at the center such that projection effects are minimized. Progress has recently been made with optical cluster surveys, in particular due to the much larger number statistics (e.g. Rozo et al. 2010, Rozo \& Rykoff 2014). We have also seen major progress with large surveys in the millimeter regime using the Sunyaev- 
Zel'dovich effect (Reichardt et al. 2013, Hasselfield et al. 2013, de Haan et al. 2016, PLANCK-Collaboration, 2016a, 2016b, Bleem et al. 2016). The cluster detection at these wavelengths is also based on the signature of the hot intracluster medium in clusters, with a signal less affected by cluster cooling cores (e.g. Motl et al. 2005).

The NORAS project is based on the ROSAT All-Sky Survey (RASS, Trümper 1993) which is the only full sky survey conducted with an imaging X-ray telescope. In the past we have already used the RASS for the construction of the cluster catalogues of the NORAS I project. While NORAS I was as a first step focussed on the identification of galaxy clusters among the RASS X-ray sources showing a significant extent, the complementary REFLEX I sample in the southern sky was strictly constructed as a flux-limited cluster sample. A major extension of the REFLEX I sample, which roughly doubles the number of clusters, REFLEX II (Böhringer et al. 2013), was recently completed. It is by far the largest, high-quality sample of X-ray selected galaxy clusters. Already the quality of the REFLEX I sample has enabled us to perform a number of cosmological studies. We demonstrated that it can provide reliable measures of the large-scale structure (Collins et al. 2000, Schuecker et al. 2001), constraining cosmological parameters (Schuecker et al. 2003a, b; Böhringer 2011) which were in good agreement with subsequently published results from WMAP (e.g. Komatsu et al. 2011). The improved quality and statistics of REFLEX II provided refined cosmological constraints (Böhringer et al. 2014), limits on the mass of neutrinos (Böhringer \& Chon 2015), and confirmed the statistical biasing concept of galaxy clusters with a precise measurement of the power spectrum of their spatial distribution as a function of their mass (Balaguera-Antolinez et al. 2011, 2012). The REFLEX data have also been used for statistical studies of cluster properties and scaling relations involving studies of statistical subsamples of REFLEX (e.g. Ortiz-Gil et al., 2004, Kerscher et al. 2001, Böhringer et al. 2007, Croston et al. 2008, Pratt et al. 2009, 2010, Arnaud et al. 2010, Böhringer et al. 2010). We also constructed a catalogue of superclusters from the latest version of the REFLEX catalogue comprising 164 superclusters including close pairs of clusters (Chon et al. 2013). We used the established statistics of this supercluster sample to arrive at a more physically motivated and quantitative definition of superclusters, by introducing a new class of objects which we call superstes-clusters (where superstes is the Latin word for survivor), which denotes those large-scale structures that will survive the cosmic expansion and collapse in the future in a $\Lambda \mathrm{CDM}$ cosmology (Chon et al. 2015). REFLEX II also allowed us to explore the matter density distribution in the local Universe in the southern sky (Böhringer et al. 2015).

During the work on the REFLEX and NORAS projects, earlier subsamples of clusters from these surveys were published in Ebeling et al. (1996, 1997, 1998), De Grandi et al. (1999), Cruddace et al. (2002). Further cluster samples based on the RASS comprise Ebeling et al. (2001, 2002).

In this paper we describe the extension of the NORAS survey to bring it to the same depth, completeness, and quality as REFLEX II. The two combined surveys, REFLEX II and NORAS II, that we termed CLASSIX (Cosmic Large-Scale Structure in X-ray) survey (Böhringer et al. 2016), now describe a homogeneous all-sky cluster sample which just leaves out the so-called zone-ofavoidance ( \pm 20 degrees around the Galactic plane). The combined survey was recently used to obtain statistical constraints on magnetic fields in galaxy clusters through a correlation of rotation measures of distant polarised radio sources with the location of X-ray luminous galaxy clusters in the sky (Böhringer et al. 2016).

The NORAS II survey now reaches a flux limit of $1.8 \times 10^{-12} \mathrm{erg} \mathrm{s}^{-1} \mathrm{~cm}^{-2}$ in the $0.1-2.4 \mathrm{keV}$ band. Redshifts have been obtained for all of the 860 clusters in the NORAS II catalogue, except for 25 clusters for which observing campaigns are scheduled. Thus with $3 \%$ missing redshifts we can already obtain a very good view of the properties of the NORAS II cluster sample and obtain some first results, which we present in this paper.

The paper is organised as follows. In chapter 2 we describe the survey properties and in section 3 the identification procedure and the properties of the sources. Section 4 explains the derivation of the selection function and section 5 provides some statistics on the survey. In section 6 we present the X-ray luminosity function and in section 7 we show first results on constraints of cosmological parameters from the NORAS II survey. Section 8 provides a summary and the conclusions.

For the derivation of distance dependent parameters we use a geometrically flat $\Lambda$-cosmological model with $\Omega_{m}=0.3$ and $h_{70}=H_{0} / 70 \mathrm{~km} \mathrm{~s}^{-1} \mathrm{Mpc}^{-1}=1$. All uncertainties without further specifications refer to $1 \sigma$ confidence limits.

\section{SURVEY PROPERTIES}

The NORAS II survey covers the sky region north of the equator outside the band of the Milky Way $\left(\left|b_{I I}\right| \geq 20\right.$ deg.) We also excise a region around the nearby Virgo cluster of galaxies which extends over several degrees on the sky, where the detection of background clusters is hampered by bright X-ray emission. This region is bounded in right ascension by $R A=185-191.25^{\circ}$ and in declination by $D E C=6-15^{\circ}$ (an area of $\sim 53$ $\left.\operatorname{deg}^{2}\right)$.With this excision the survey area covers 4.18 steradian (13519 $\mathrm{deg}^{2}$, a fraction of $32.7 \%$ of the sky). NORAS II is based on the RASS product RASS III (Voges et al. 1999) which was also used for REFLEX II. The 
NORAS II survey was constructed in an identical way as REFLEX II with a nominal flux limit of $1.8 \times 10^{-12} \mathrm{erg} \mathrm{s}^{-1}$ $\mathrm{cm}^{-2}$. The northern sky has on average slightly larger exposures than the southern sky, due to the more frequent shut down times in the particle belts of the South Atlantic Anomaly affecting the southern sky. Fig. 1 shows a comparison of the exposure time distribution for NORAS II in comparison to REFLEX II. Since the construction of the NORAS I survey was based on the detection of clusters as extended X-ray sources in an early version of the RASS data set and since the detection of a significant extent in published RASS results was imperfect, NORAS I was not statistically complete. Therefore NORAS II is now the first statistically well described cluster sample for the northern ROSAT sky.

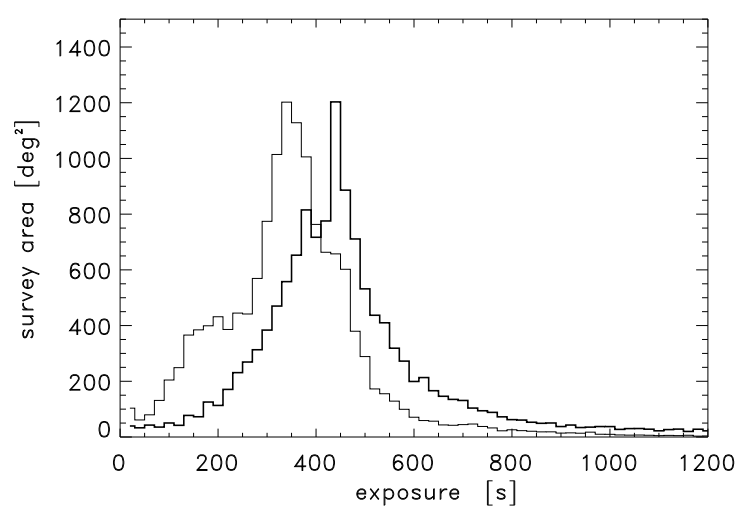

Figure 1. Exposure time distribution of the NORAS II survey (thick line) in comparison to that of the REFLEX II survey (thin line). Due to the smaller number of detector shut-off times in the radiation belts in the Northern sky, the exposure coverage is better than in the South.

The source selection was based on an intermediate stage source catalogue produced during the production of the RASS III data release (Voges et al. 1999). 54,286 sources of this catalogue fall into the NORAS II survey region. As in the case of REFLEX II, we reprocessed all these sources with the growth curve analysis (GCA) method, because we know that the standard analysis tuned for point sources tends to underestimate the flux of extended X-ray emission (Böhringer et al. 2000). A preliminary flux limit of $1.4 \times 10^{-12} \mathrm{erg} \mathrm{s}^{-1} \mathrm{~cm}^{-2}$ was then imposed on the reprocessed source catalogue yielding 3902 sources which were further inspected in detail, as described below. The nominal flux limit of $F_{n} \geq 1.8 \times 10^{-12} \mathrm{erg} \mathrm{s}^{-1} \mathrm{~cm}^{-2}$ has been imposed after visual inspection, to allow sources to be recovered in case the automatic flux measurement was not perfect.

\section{X-RAY PARAMETERS AND SOURCE IDENTIFICATION}

The X-ray fluxes and luminosities of the sources are based on the source count rates determined with the growth curve analysis method, which is described in detail in Böhringer et al. $(2000,2001)$. The final values for flux and luminosity in a well defined aperture are derived in three steps, which are described in detail in Böhringer et al. (2013) and illustrated with a flow diagram in their Figure 4 . The three steps, which are briefly described in the following, involve the determination of (i) a preliminary, nominal flux without the knowledge of the source redshift, (ii) a corrected flux taking the redshift information into account, and (iii) a flux in a well defined aperture.

- nominal flux: The count rate was measured with the GCA method in the energy band defined by ROSAT PSPC channels 52 to 201 ( $\sim 0.5$ to $2 \mathrm{keV}$ ), where the signal to noise is highest, and converted to a "nominal" flux, $F_{n}$, in the broad ROSAT band, 0.1 to $2.4 \mathrm{keV}$. The aperture radius, $r_{\text {out }}$, for the count rate determination is defined by the radius where the cumulative background-subtracted count rate (growth curve) reaches a plateau without further significant growth (for details see Böhringer et al. 2000, 2001). The conversion from count rate to unabsorbed nominal X-ray flux, $F_{n}$, is performed by assuming a thermal plasma spectrum for a temperature of $5 \mathrm{keV}$, a metallicity of 0.3 of the solar value (Anders \& Grevesse 1989), a redshift of zero, and an interstellar hydrogen column density given for the cluster line-of-sight in the compilation by Dickey \& Lockman (1990). ${ }^{1}$ The value of $F_{n}$ is used to make the flux cut independent of any redshift information (since the redshift is not available for all objects at the start of the survey).

- corrected flux and luminosity: With known redshift, an improved flux calculation is performed to obtain the unabsorbed X-ray flux, $F_{x}$. For this conversion we use the cluster redshift and assume a temperature for the intracluster medium, that we derive from the $\mathrm{X}$-ray luminosity-temperature relation

$$
T=3.31 L_{X}^{0.332} h_{70}^{0.666},
$$

where $T$ is in $\mathrm{keV}$ and $L_{X}$ is measured inside $r_{500}{ }^{2}$ in units of $10^{44} \mathrm{erg} \mathrm{s}^{-1}$. The $L_{X}-T$ relation is

1 We have compared the results obtained with the interstellar hydrogen column density compilation by Dickey \& Lockman (1990) with those of the Bonn-Leiden-Argentine $21 \mathrm{~cm}$ survey (Kalberla et al. 2005) and found that the difference is of the order of a percent. Because our survey has been constructed with a flux cut based on the earlier results, we keep the older hydrogen column density values for consistency of the cluster parameters with the selection function.

${ }^{2} r_{500}$ is used for the fiducial outer radius of the clusters, defined 
taken from our REXCESS study (Pratt et al. 2009, Böhringer et al. 2012). Motivated by the results of Reichert et al. (2011), we assume that the $L_{X}$ - $T$ relation has no redshift dependence. This correction is less than $5 \%$ for sources with an X-ray luminosity above $4 \times 10^{43} \mathrm{erg} \mathrm{s}^{-1}$. The use of a redshifted spectrum in the folding with the instrument response is equivalent to a $\mathrm{K}$-correction in optical astronomy. Tables for the conversion from count rate to flux and for the "K-correction" are given in Böhringer et al. (2013) and are available on-line ${ }^{3}$

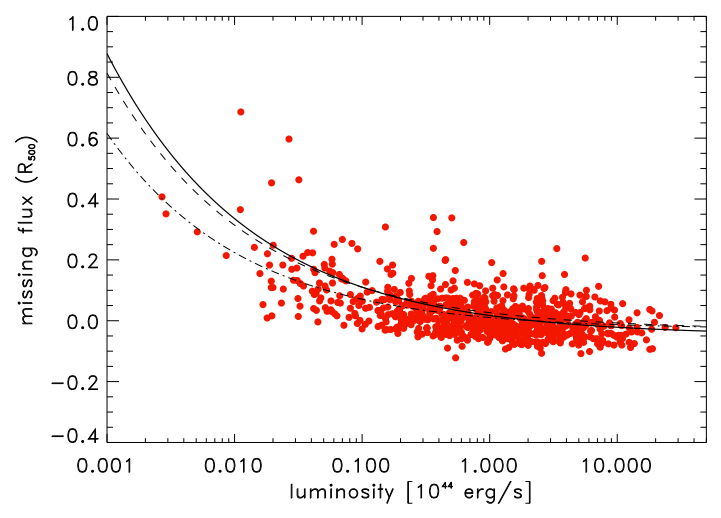

Figure 2. Estimated missing flux as a function of X-ray luminosity for the clusters in the NORAS II sample. The effect of the ROSAT Survey point spread function has been included. The corrections are largest for low luminosity objects. The solid line shows the best fitting power law function with linear offset (Eq. 2). The dashed line shows the corresponding values for REFLEX II. The dotted-dashed line shows a fit, where 21 outliers have been excised, to illustrate the large influence of the extreme outliers on the fit.

- aperture flux and luminosity: For general use we determine the X-ray luminosity inside an aperture radius of $r_{500}$. The procedure to convert the luminosities measured in the aperture radius $r_{\text {out }}$ into luminosities inside $r_{500}$ is described in detail in Böhringer et al. (2013). The correction requires two pieces of information. $r_{500}$ is calculated from the cluster mass, which is in turn estimated from the $L_{X}-M$ scaling relation (Eq. 5, see also Böhringer et al. 2014). In addition we have to correct for the effect that, with the limited angular resolution of the survey, flux originally inside $r_{500}$ is spread outside the aperture. The half power radius of the RASS point spread function is almost

as the radius inside which the mean mass density of the cluster is 500 times the critical density of the Universe at the cluster redshift.

3 They are available on CDS via anonymous ftp at cdsarc.ustrasbg.fr and on: http://www.mpe.mpe.de/ hxb/REFLEX
1.5 arcmin, and therefore this effect cannot be neglected.

We use the NORAS II cluster catalogue to illustrate the relevance of this aperture flux correction in Fig. 2. We find that the missing flux depends most strongly on the cluster X-ray luminosity, as shown in the Figure. The correction is most significant for the smaller, less luminous systems. Due to their low surface brightness, we detect them with a smaller extent. For clusters with luminosities above $10^{43} \mathrm{erg} \mathrm{s}^{-1}$, the mean correction is smaller than $2 \%$. Fig. 2 also shows a functional fit of the average correction with the following form:

$$
f_{\text {miss }}=0.0677 \times L_{X, 500}^{-0.379}-0.0504 .
$$

The result is almost identical to that obtained for the REFLEX II survey as shown in Fig. 2. We have also fitted the data excluding the 21 most extreme outliers, which resulted in a fit with a much lower reduced $\chi^{2}$ (0.94 instead of 2.6) with the result $f_{\text {miss }}=0.0387 \times L_{X, 500}^{-0.407}-0.0282$. It is shown to illustrate the influence of a few extreme data points on the fit. We are using the actual missing flux corrections, but not this fit in our further analysis and therefore this problem does not affect our results.

- To model the cluster survey it is important to know the uncertainty in the flux and luminosity determination. The main source of flux uncertainty is the Poisson statistics of the source photons. Thus it is not surprising that the uncertainty shows the clearest dependency on the number of source photons detected. We show this relation in Fig. 3, where we replaced the photon number by the parameter of the product of flux and exposure. The function shows almost a square root dependence. It can be expressed by:

$$
f_{\text {err }}=588.5 \times\left(F_{X} \times \text { exposure }\right)^{-0.504},
$$

where $F_{X}$ is given in units of $10^{-12} \mathrm{erg} \mathrm{s}^{-1} \mathrm{~cm}^{-2}$ and the exposure in sec. This result is practically identical to the one obtained for REFLEX II as shown in Fig. 3 .

The mean uncertainty of the flux is $17 \%$. Some data points that appear up-scattered from the average relation in Fig. 3 include those cases where sources have been deblended and where the error increases due to the additional uncertainty in deblending. 


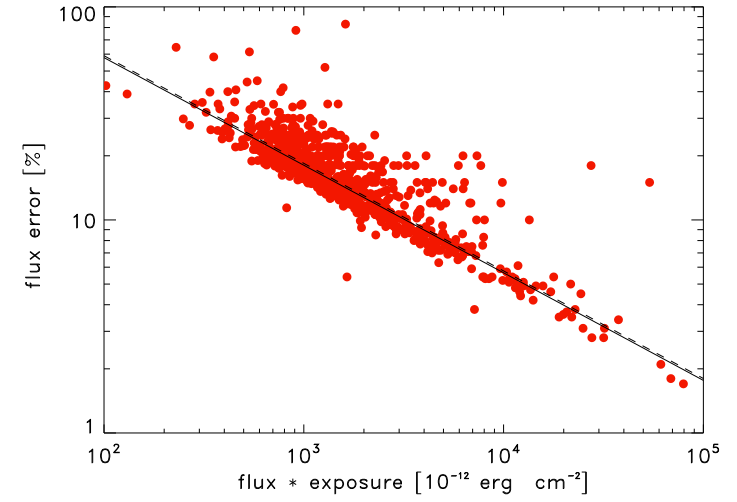

Figure 3. Uncertainty of the flux determination for the NORAS II clusters as a function of the product of flux and exposure time. This functional form is close to pure Poisson statistics with a dependence on the square of the photon number. The solid line shows the best power law fit (Eq. 3). The dashed line shows the corresponding result for REFLEX II.

We make use of two further parameters which characterise the X-ray sources and which are delivered by our GCA pipeline: the spectral hardness ratio and the extent of the source. The hardness ratio, $H R$, is defined by $H R=\frac{H-S}{H+S}$ where $H$ is the hard and $S$ the soft band source count rate. Both rates are determined using the same aperture radius. The bands are defined on the basis of the ROSAT PSPC energy channels: from 10 to 40 (for the soft band) and from 52 to 201 (for the hard band). These intervals correspond roughly to the energy bands $0.1-0.4 \mathrm{keV}$ and 0.5 to $2.0 \mathrm{keV}$, respectively. For galaxy clusters we can calculate the expected hardness ratios from the spectral properties for given temperature, redshift, and interstellar hydrogen column density, $n_{H}$. For clusters with temperatures above $2 \mathrm{keV}$ the hardness ratio depends mostly on the interstellar column density. Using the observed $n_{H}$ from Dickey and Lockman (1990) we can determine the expected hardness ratio approximately (assuming a temperature of $5 \mathrm{keV}$ and a metallicity of 0.3 solar) and apply this parameter in our cluster identification process.

We make use of the hardness ratio in the following way. We compare the expected HR values to the observed ones and their estimated uncertainties. We illustrate the usefulness of this parameter in Fig. 4, where we compare the hardness ratio deviations for different types of X-ray sources. ${ }^{4}$ Galaxy clusters populate the high end of the hardness ratio distribution. The stars are mostly softer than the clusters and the majority of the AGN sources can be distinguished from clusters as been too soft. We

\footnotetext{
${ }^{4}$ The stellar and AGN X-ray sources used here have been compiled during our source identification process from X-ray sources above the flux limit with a safe identification as stars or AGN from the literature.
}

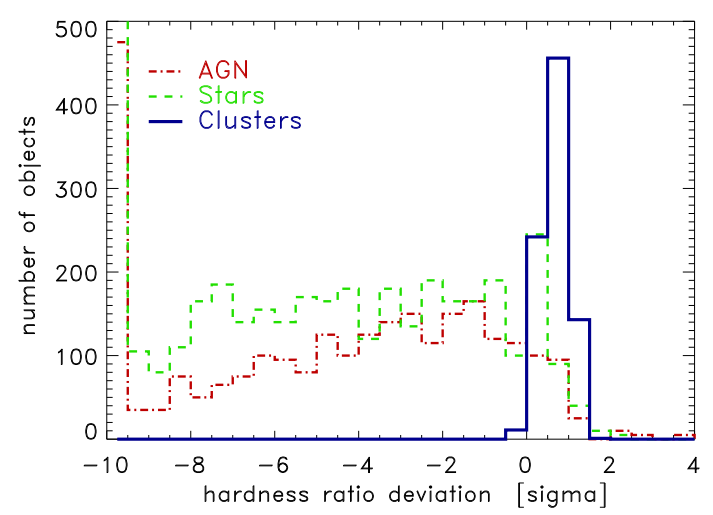

Figure 4. Hardness ratio parameter distribution of various classes of X-ray sources: galaxy clusters, AGN, and stars. The $\mathrm{X}$-axis shows the deviation of the observed hardness ratio from the expectation for a galaxy cluster given a temperature of $5 \mathrm{keV}$ and the interstellar absorption in the line-of-sight as taken from Dickey \& Lockman (1990). The unit of the deviation is given in $\sigma$-values of the hardness ratio uncertainty. A substantial fraction of the non-cluster sources can be distinguished by their hardness ratio from clusters.

conservatively mark an X-ray source to be too soft to originate from a cluster, when the observed value is more than $3 \sigma$ away from the expectation.

Compared to the majority of the sources which are mostly stars and AGN, cluster sources are extended. Therefore the finding of a source extent is a strong indication for a cluster candidate. We cannot rule out a cluster nature of a source, however, if we do not find an extent, since not all X-ray cluster sources at higher redshift can be spatially resolved in the ROSAT Survey. We search for a source extent by applying a Kolmogorov-Smirnov (KS) test to estimate the probability that the photon distribution of the source is consistent with a point source. A probability less than 0.01 is taken as sign of a significant source extent. KS tests applied to X-ray sources with an identification as stars or AGN return a false classification rate of not being consistent with a point source with a probability less than 0.01 on the $5 \%$ level.

The likely cluster candidates were selected from the flux limited list of 3902 X-ray sources based on our experience gained with REFLEX, where identical selection criteria had been used. First of all, we cannot rely on the detection of extended X-ray emission for the selection, since not all clusters are resolved in the survey with the survey PSF. For this reason we considered all X-ray sources and took the following information into account: the X-ray properties of the sources determined from the RASS, firm object identifications listed in $\mathrm{NED}^{5}$ at the

${ }^{5}$ see http://ned.ipac.caltech.edu/ 


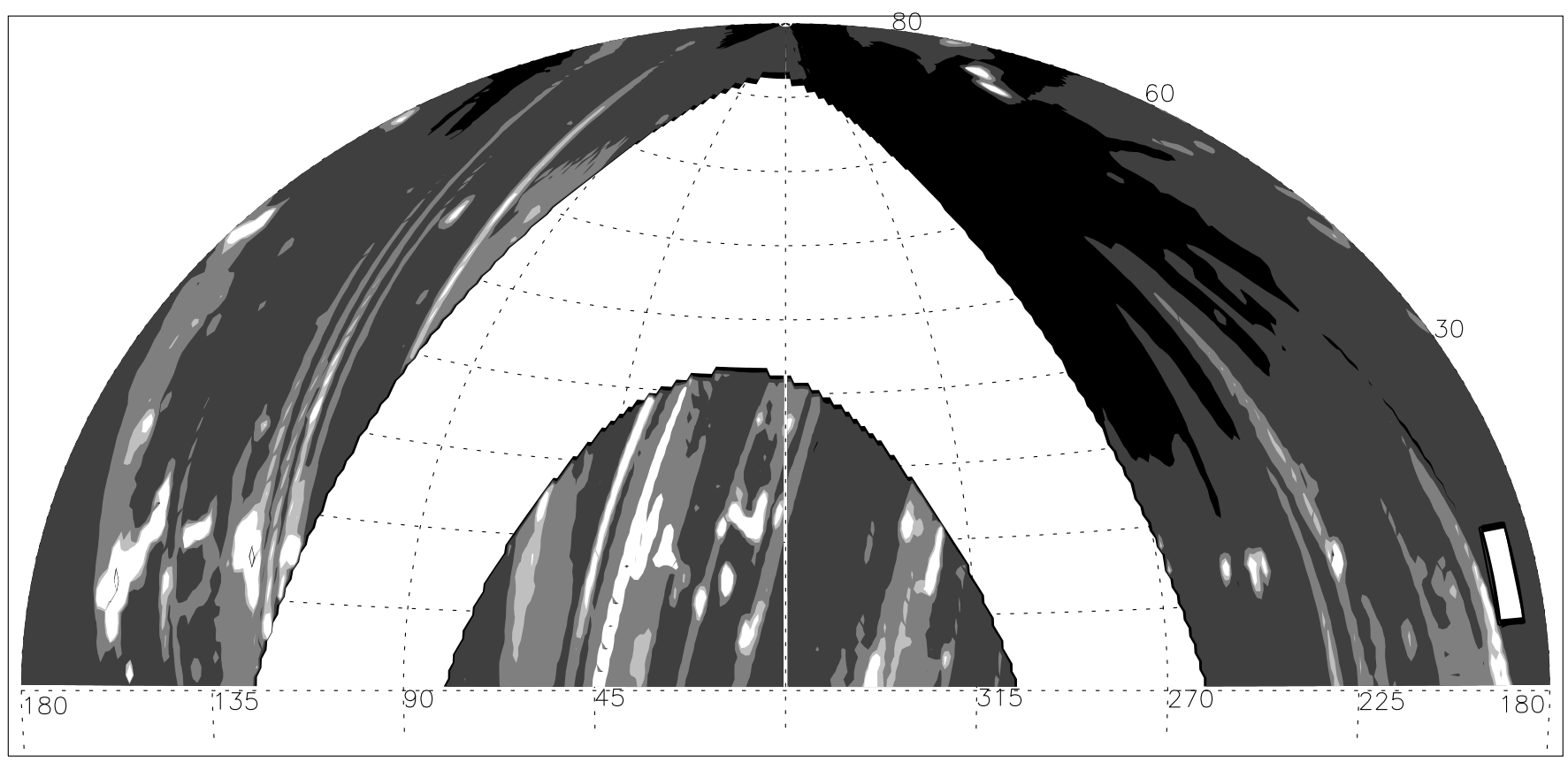

Figure 5. Sensitivity map of RASS III for the region of the NORAS II survey in an equatorial coordinate system (J2000). We show the number of photons detected for a source at the nominal flux limit using five levels of increasing grey scale for photon numbers $<15,15-20,20-30,30-60$, and $>60$, respectively. The white box shows the Virgo cluster region (RA: 185 - 191.25 , DEC: 6 - 15 ${ }^{\circ}$ ). The data of the map are made available on-line as explained in Appendix B.

source positions, digital sky images obtained from $\mathrm{DSS}^{6}$, and any relevant information from the literature. An inspection of DSS images with superposed contour images of the X-ray surface brightness turned out to be very helpful. For X-ray sources already clearly identified in the literature we adopt this classification if we have no doubt about it after an inspection of the observational information.

In Böhringer et al. (2013), which described the construction of the REFLEX II survey, we provided a detailed description of the selection scheme including an overview by means of a flow chart of the selection process (see their Fig. 9). To discard an X-ray source from the cluster candidate list in a first step, we typically use at least two negative criteria: (i) the source is too soft and is consistent with being a point source, (ii) the source is a point source and coincident with a bright star, known galactic X-ray source, or AGN, (iii) the source is flagged as extended and coincident with a nearby galaxy, galactic HII region, or supernova remnant, (iv) the source is flagged as extended but best explained by multiple sources with a hardness ratio or a morphology inconsistent with being a cluster. For some point sources with spectral properties not inconsistent with a cluster, but no trace of a galaxy concentration on the optical images, we are left with no

${ }^{6}$ see http://archive.stsci.edu/dss/ classification. In case there is any weak indication of some faint galaxies at the X-ray center or for completely blank fields we have taken deeper images as part of our observation runs. Inspecting more than 40 such borderline cases that had not been flagged as promising candidates we did not find a cluster. Since most promising fields have been targeted, we are convinced that we have reached a high completeness in our cluster identification of the flux limited source list. On the positive selection side we could be more generous to include weak cluster candidates since in the follow-up spectroscopic identification, which is described below, the false clusters are revealed.

In some cases we found that the cluster X-ray emission was contaminated by X-rays from point sources. In this case we did the best effort to deblend the point source from the cluster emission and added the estimated uncertainty of the deblending procedure to the flux error. Before deciding to deblend, it is important to distinguish between truly contaminating point sources and cluster substructure, where the latter is considered part of the genuine cluster emission. We therefore deblended only sources which can be recognised as non-cluster emission by having a clearly separable point source nature, by showing an obvious counterpart for the X-ray source, or showing a distinctly different local hardness ratio. A nice 
example for the latter case is shown in Fig. 3 in Chon \& Böhringer (2012). In another set of sources inspected during the source identification process, there was either a previously identified group or cluster of galaxies near the $\mathrm{X}$-ray position or a cluster was well visible in the digital sky images, but it was obvious that the $\mathrm{X}$-ray emission was likely dominated by an AGN, while the possible cluster emission fell below the flux threshold of the survey. In these cases we removed the sources from the catalogue, and plan to give a list of them with the publication of the cluster catalogue, as done for REFLEX I (Böhringer et al. 2004).

The confirmation of the cluster's nature and redshift measurement by follow-up spectroscopic observations were described in Guzzo et al. (2009) for REFLEX I and in Chon and Böhringer (2012) for REFLEX II and they apply in a similar way to the NORAS project. For the NORAS II survey, observations were performed mainly at the German-Spanish Calar Alto Observatory at the 3.5 and $2.2 \mathrm{~m}$ telescopes. For NORAS I we also conducted observations at Mt. Hopkins (Böhringer et al. 2000). Whenever possible, we used multi-slit spectroscopy, which typically provided about 7 cluster galaxy redshifts. To make the observing runs most efficient, in particular at the smaller telescopes, we also used long slit observations with two or three targets per slit. For these cases it was important to include the BCG as one of the targets, which helped very much to get a unique cluster redshift. The typical galaxy redshift uncertainty is 50 $60 \mathrm{~km} / \mathrm{s}$ for our follow-up observations.

From the selection and identification of the X-ray source list we obtained a catalogue of currently 853 galaxy clusters, with 7 additional clusters in the Virgo region. For this sample we have not yet applied a lower limit in the detected number of source photons of 20. 21 clusters in the sample have less than 20 and 7 less than 15 source photons.

\section{SURVEY SELECTION FUNCTION}

To provide a model of the survey for the further data analysis the survey selection function has to be known. To characterise the survey sensitivity of NORAS II for each position in the sky, we calculate the flux limit per photon based on the exposure time of RASS III and the interstellar column density taken from Dickey \& Lockmann (1990). For the count rate to flux conversion we use the same conversion calculation as used in section 2 for the determination of the nominal source fluxes, with the same assumptions (ICM temperature of $5 \mathrm{keV}$ and metallicity of 0.3 solar). We call this the sensitivity map of the survey, and show it in Fig. 5. The map shows the number of detectable photons for sources at the nominal flux limit in pixels with a resolution of one square degree. There are stripes of low sensitivity, which are due to low exposure regions suffering from shut down times of the ROSAT detectors during the crossing of the radiation belts. The NORAS map has fewer of these low exposure areas than the corresponding southern sky map. This results in a higher average exposure of the NORAS region compared to the REFLEX survey. The deepest exposure is at the North Ecliptic Pole region at $18^{h}$ and $+66^{\circ} 33^{\prime}$ 38.5 ".

In the next step we calculate the nominal flux limit for the survey as a function of sky position imposing a limit for the minimum number of source counts for a robust detection. Different values for this limit could be chosen depending on the requirements for the quality of the source properties. We have chosen a minimum limit of 20 source photons for several reasons. Decreasing this photon number, the flux and hardness ratio uncertainties increase relatively fast. Also the region of the survey, where the nominal flux limit is not reached, increases. While these uncertainties can in principle be taken care of, accurate estimates of the uncertainties become increasingly difficult below a photon number of 20 . On the other hand few sources are lost by imposing this cut, as described above. Therefore we lose in general more than we gain by lowering this limit. The cumulative sky area covered by the survey as a function of the nominal flux limit for a minimum detection of 20 photons is shown in Fig. 6. We also provide the corresponding curve for REFLEX II. We note that over $92.2 \%$ of the sky area the nominal flux limit of $1.8 \times 10^{-12} \mathrm{erg} \mathrm{s}^{-1} \mathrm{~cm}^{-2}$ is reached. Only the smaller remaining part has a higher limiting flux. The figure also shows that in the northern sky the area in which the nominal flux-limit is reached is larger compared to that of the REFLEX survey. We provide the data of the curve in numerical format in the on-line material as described in the Appendix.

The construction of the X-ray luminosity function and several cosmological studies require the knowledge of the survey selection statistics as a function of cluster luminosity. We provide this information in the form of the minimum cluster luminosity required for a detection as a function of sky position and redshift. We will refer to this multi-dimensional function as the selection mask of the survey. This mask provides for example the direct recipe to select clusters from a cosmological N-body simulation to create a mock survey sample. The mask was applied in exactly this way in our study of the REFLEX II power spectrum in Balaguera-Antolinez et al. (2011).

To determine the selection mask we calculate for each sky pixel the limiting luminosity as a function of redshift. While this calculation starts with a $5 \mathrm{keV}$ ICM spectrum for the count rate - flux conversion, we iteratively include the estimated temperature, the "K-correction" and the missing aperture flux in the calculation. The mask is tabulated for 160 redshifts out to $z=0.8$ and for 21666 


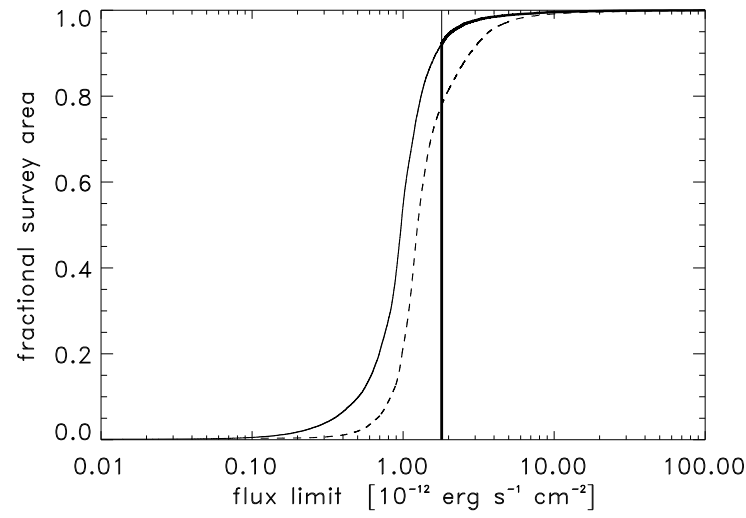

Figure 6. Effective sky coverage of the NORAS II sample. The thick line gives the effective sky area for the nominal flux limit of $1.8 \times 10^{-12} \mathrm{erg} \mathrm{s}^{-1} \mathrm{~cm}^{-2}$ and a minimum number of 20 photons per source. The solid line without the vertical cut at a flux of $1.8 \times 10^{-12} \mathrm{erg} \mathrm{s}{ }^{-1} \mathrm{~cm}^{2}$ shows the 20 photon limit. The dashed line shows the equivalent 20 photon limit for the REFLEX II Survey.

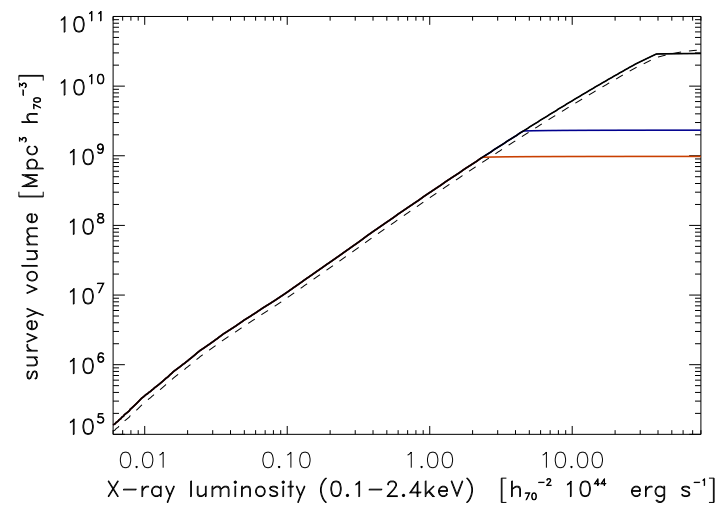

Figure 7. Effective survey volume as a function of Xray luminosity of the NORAS II survey. The solid curve shows the survey volume for a redshift limit of $z=0.8$ (upper curve), a limit of $z=0.3$ (middle curve) and a limit of $z=0.22$ (lower curve), respectively. The dashed line shows the REFLEX II survey volume for a redshift limit of $z=0.8$.

sky pixels. The data for the mask are given in the on-line material in a form further described in the Appendix.

Another useful statistic, required for example to determine the cluster X-ray luminosity function, is the effective survey volume probed by NORAS II as a function of $\mathrm{X}$-ray luminosity. We show this function in Fig. 7 for the fiducial $\Lambda \mathrm{CDM}$ cosmological model defined in the introduction. We limited the volume calculations to redshifts up to $z=0.8$ (shown as solid line) and alternatively to redshift limits of $z=0.3$ and $z=0.22$ (shown by shown by dashed lines), respectively. For a luminosity of $L_{X}=5 \times 10^{44} \mathrm{erg} \mathrm{s}^{-1}$, which is not far from $L^{\star}$ of the

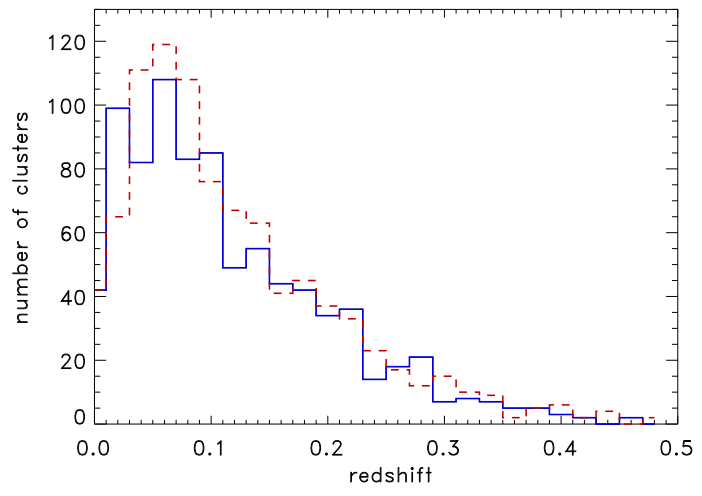

Figure 8. Redshift distribution of the NORAS II clusters (solid line). This function is compared to that of the REFLEX II sample (dashed line).

$\mathrm{X}$-ray luminosity function, we reach a survey volume of $\sim 2.5 \mathrm{Gpc}^{3}$. The redshift limit of $z=0.3$ approximately corresponds to this limiting luminosity.

\section{STATISTICAL PROPERTIES OF THE CLUSTER SAMPLE}

Fig. 8 shows the redshift distribution of the NORAS II clusters in comparison to those of REFLEX II. The redshift distributions are quite similar. The median redshift for the NORAS II sample is $z=0.102$ and the mean $z=0.125$. There is a long tail of distant clusters out to redshifts of $\sim 0.5$.

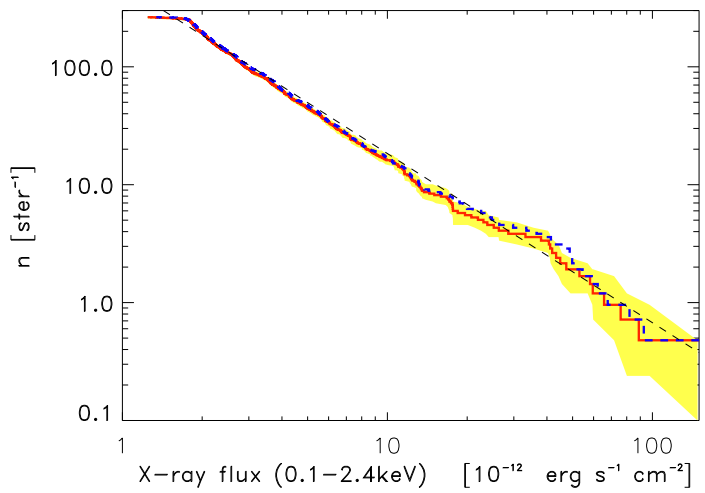

Figure 9. $\log \mathrm{N}-\log \mathrm{S}$ distribution of the clusters in the NORAS II survey. The solid line shows the function for measured fluxes and the dashed line for those of the fluxes inside $r_{500}$, corrected for missing flux. The dashed, straight line shows a fit to a power law with a $\log \mathrm{N}-\log \mathrm{S}$ slope of -1.44 . The shaded region shows the $1 \sigma$ uncertainties obtained from bootstrap simulations.

The cluster number counts per unit sky area as a function of limiting flux, the so-called $\log \mathrm{N}-\log \mathrm{S}$ distribution, for NORAS II is shown in Fig. 9. It is restricted to clusters with more than 20 photon counts. The source density is 
calculated with a sky area normalisation derived from the nominal flux, $F_{n}$, and the sensitivity map shown in Fig. 5. The flux values used in the curve are the corrected observed flux, $F_{X}$ (solid line), and the flux corrected for missing flux inside $r_{500}$ (dashed line). The best fitting slope for the observed $\log N-\log S$ function is about -1.44 , slightly steeper than the fit to the $\log \mathrm{N}-\log \mathrm{S}$ function of REFLEX II.

To estimate the statistical uncertainties of the LogN$\log \mathrm{S}$ function we performed bootstrap simulations by resampling from the observed cluster flux distribution, allowing the total number of clusters in the sample vary within its Poisson errors. We obtained $1 \sigma$ limits for the uncertainties by determining the $16 \%$ and $84 \%$ percentiles of the cluster number densities as a function of flux from 1000 simulations as shown in Fig. 9.

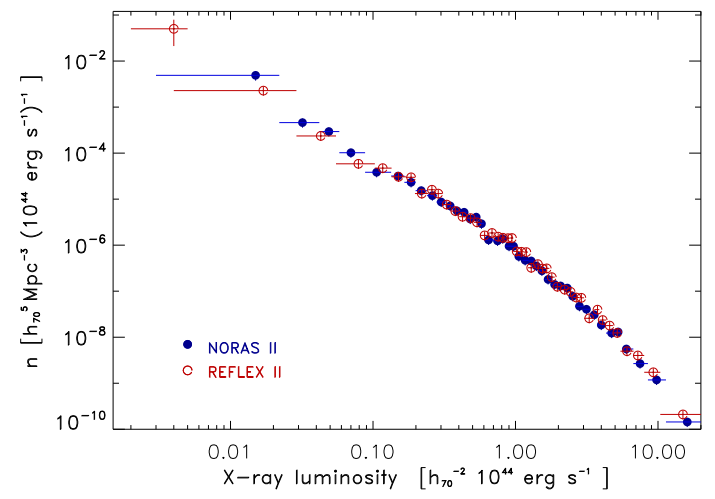

Figure 10. X-ray luminosity function for the clusters from the NORAS II survey (filled symbols). For comparison we show also the results from REFLEX II (open symbols).

\section{THE X-RAY LUMINOSITY FUNCTION}

The most important census of the population of X-ray selected clusters in the Universe is the X-ray luminosity function (XLF). This function is determined from the catalogue of clusters and the survey selection function in the form of the effective survey volume as a function of $\mathrm{X}$-ray luminosity as shown in Fig. 7 . We use a source detection count cut of minimum 20 photons for the selection. Then the binned differential X-ray luminosity function is given by

$$
\frac{d n\left(L_{X}\right)}{d L_{X}}=\frac{1}{\Delta L_{X}} \sum_{i} \frac{1}{V_{\max }\left(L_{X_{i}}\right)}
$$

where $V_{\max }$ is the effective detection volume, $\Delta L_{X}$ is the width of the luminosity bin, and the sum includes all clusters in the bin. Fig. 10 shows the XLF derived for a binning with 20 clusters per bin, except for the bin at the lowest X-ray luminosity which contains only 18 clusters. The luminosity values used in the construction of the XLF are the luminosities inside $r_{500}$, corrected for missing flux in the $0.1-2.4 \mathrm{keV}$ rest frame band. The sub-sample used for the analysis covers the redshift range $z=0$ to 0.4 . The error bars for the XLF given in the figure are the Poisson uncertainties for the number of clusters per bin. The XLF covers more than three orders of magnitude in luminosity and comprises objects from small groups to the largest clusters we know.

In the figure we also compare the NORAS II XLF to that of REFLEX II and find excellent agreement within the Poisson uncertainties. We do not expect cosmic variance to play a significant role here, except for the lowest luminosity bin, as shown in Böhringer et al. (2002). The redshift range used for the construction of the XLF is rather large, $z=0$ to 0.4 , and thus one may wonder about the effects of redshift evolution. For the case of REFLEX II we have looked into the evolution of the XLF in detail and found no significant effect in this redshift range (Böhringer et al. 2014). We can attribute the lack of observed evolution to the fact that an expected mild evolution in the mass function is compensated by an evolution of the mass - X-ray luminosity relation.

\section{CONSTRAINING COSMOLOGICAL PARAMETERS}

The most immediate application of these results to cosmology is the use of the cluster abundance encoded in the XLF to constrain cosmological parameters by a comparison of the observational data with theoretical predictions for various cosmological models. We apply here to the NORAS II data set exactly the same approach that we used in Böhringer et al. (2014) for the REFLEX II sample. The only difference is the calculation of the large-scale structure matter power spectrum by means of the program CAMB (Lewis et al. 2000) ${ }^{7}$ instead of the power spectrum by Eisenstein \& Hu (1998) used previously. This change makes a difference in the results of less than one percent. In brief the modelling of the cluster luminosity function involves the following steps. We calculate theoretical predictions for the cluster mass function from structure formation theory including the mass function formulas by Tinker et al. (2008). We use the following X-ray luminosity - mass relation (also used in Böhringer et al. 2014)

$L_{500}(0.1-2.4 \mathrm{keV})=0.1175 M_{200}^{\alpha_{s l}} h^{\alpha_{s l}-2} \quad E(z)^{\alpha_{s l}}$

to convert the mass function to a cluster XLF taking account of the uncertainties and the scatter of the relation. This relation is based on observations and chosen

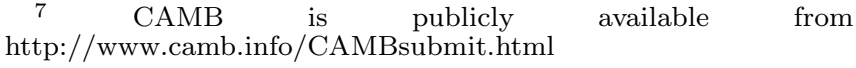


Table 1. Default cosmological model parameters.

\begin{tabular}{l|lr}
\hline \hline Parameter & \multicolumn{1}{c}{ Explanation } & \multicolumn{2}{c}{ Value } \\
\multicolumn{1}{c}{$(1)$} & \multicolumn{1}{c}{$(2)$} & $(3)$ \\
\hline$h_{100}$ & Hubble parameter & 0.70 \\
$\Omega_{b}$ & baryon density & 0.045 \\
$n_{s}$ & Primodial P(k) slope & 0.96 \\
$\alpha_{s l}$ & $L_{X}-M$ relation slope & $1.51 \pm 7 \%(1 \sigma)$ \\
$n_{0}$ & $L_{X}-M$ relation norm. & $0.1175 \pm 14 \%(1 \sigma)$ \\
mass bias & X-ray mass underestimation & 0.1 \\
\hline
\end{tabular}

Note-We use a flat $\Lambda$ CDM cosmological model with the parameters given in the Table.

such that it is consistent with our results from the REXCESS study in Pratt et al. (2009), with the work of Vikhlinin et al. (2009) and with our earlier results reported in Reiprich \& Böhringer (2002). The resulting, predicted XLF is compared to the observations of NORAS II by means of a maximum likelihood method. We allow for measurement uncertainties for the X-ray fluxes and luminosities of $20 \%$.

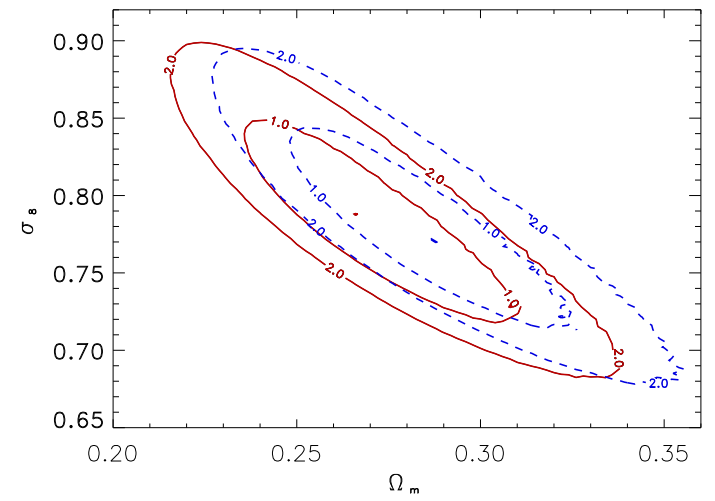

Figure 11. Constraints on the cosmological parameters $\Omega_{m}$ and $\sigma_{8}$ obtained from the NORAS II cluster sample (solid lines, 1 and $2 \sigma$ contours) compared to the results from the REFLEX II survey (dashed lines).

We concentrate here on the constraints of the cosmological parameters $\Omega_{m}$ and $\sigma_{8}$, to which the galaxy cluster XLF is most sensitive. The other cosmological parameters were fixed to the same values as in our cosmological analysis of REFLEX II (Böhringer et al. 2014) with negligible neutrino mass. The cosmological and scaling relation parameters we use are summarised in Table 1.

As in Böhringer et al. (2014) we use a generous marginalisation over the uncertainties in the scaling relation given in Eq. 1, with errors on the $L_{X}-M$ relation slope of $\pm 7 \%$, and for the normalisation of $14 \%$, taken as $1 \sigma$ uncertainties. The uncertainty in the normalisation is equivalent to a bias in the mass calibration for the relation. We allow for a scatter in the relation of $30 \%$. In addition we also assume that the mass calibration, which is based on hydrostatic mass determinations using X-ray data, is biased low by $10 \%$. For a detailed discussion of the effect of these uncertainties and the justification for their values see Böhringer et al. (2014).

Fig. 11 shows the constraints we obtain for the two cosmological parameters $\Omega_{m}$ and $\sigma_{8}$. We compare the NORAS II results in the figure to those of REFLEX II and we find excellent agreement within the statistical uncertainties. We will not discuss the cosmological implications of these results here, since this can be found in our previous publications Böhringer et al. (2014, 2015) and Böhringer \& Chon (2016).

\section{DISCUSSION}

Most of the systematics that affect these studies has been discussed in Böhringer et al. (2014). One aspect we investigate here in more detail than what has been done previously is the question how the results are affected by an incompleteness of the cluster sample, since we are still missing $3 \%$ of the redshifts for the NORAS II clusters, which are therefore not included in the cosmological analysis. Thus we have repeated the above exercise of cosmological parameter constraints with two cluster samples, where the cluster catalogue for sample A has been reduced to $80 \%$ of the original catalogue (by excluding the last $20 \%$ of the clusters in right ascension order). For sample B the first $30 \%$ of the clusters in right ascension order have been counted twice. The parameter constraints for the original sample and for the two manipulated samples are shown in Fig. 12. The changes of the results shown are much larger than what we expect for any realistic incompleteness, to illustrate the effect more clearly.

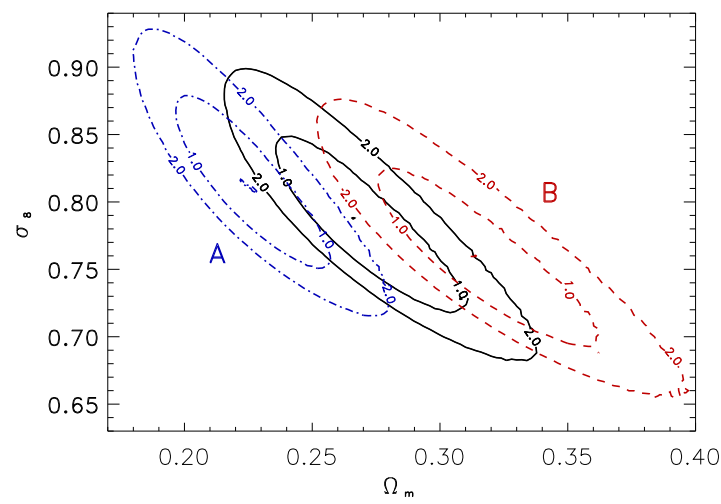

Figure 12. Effect of the sample completeness on the cosmological parameter constraints. We show the variation of the marginalised constraints on $\Omega_{m}$ and $\sigma_{8}$ for a sample depleted to $80 \%$ (A), a nominal sample $(100 \%$, solid lines) and an artificially enriched sample $(130 \%, \mathrm{~B})$ of the NORAS II survey. 
The dependence of the results on the sample size can be roughly parameterised by $\frac{d \log \Omega_{m}}{\operatorname{dlog} S} \sim 0.7$ and $\frac{d \log \sigma_{8}}{d \log S} \sim$ -0.15 . This is only valid close to the nominal value as the dependence is not linear. For NORAS II, where $3 \%$ of the redshifts are still missing, this would imply an increase of $\Omega_{m}$ and $\sigma_{8}$ for the full sample of about $2 \%$ and $0.5 \%$, respectively. Overall we expect that the sample could be incomplete in the cluster detection by up to about $6 \%$, which would introduce a bias of about $4 \%$ and $1 \%$, respectively, which is still well within the statistical uncertainties.

This test was performed under the assumption that the incompleteness is statistically random. However, the clusters remaining without redshifts so far may have highly biased properties - at least most of them are close to the flux limit and not nearby. In the Appendix we therefore also explore the effect when the missing clusters have preferentially low or high X-ray luminosities. The results shows that the incompleteness effect shifts the results in these two cases in opposite directions. Therefore it is dangerous to make any correction for the incompleteness if we do not know the properties of the clusters with missing redshifts. For this reason we have not used the results shown in Fig. 11 to apply a correction to our cosmological results.

\section{SUMMARY AND CONCLUSION}

One of the major objectives in compiling the NORAS II cluster sample was to make the northern RASS cluster sample compatible with REFLEX II. Therefore we took care to construct the sample with the same selection parameters and aimed for the same quality as for the REFLEX II Survey. The good agreement of all the results on the survey statistics and the cosmological parameters between the two surveys shows that we have reached this goal. Therefore we can now combine the samples for the two hemispheres to build a homogeneous all-sky cluster sample from the ROSAT Survey outside the zone-ofavoidance. This is the largest and best defined sample of X-ray luminous clusters in the Universe. It comprises 1722 clusters in a sky area of $\sim 8.25$ ster. We have used this combined survey already recently to study the statistics of rotation measures of background radio sources in the line-of-sight of the sample clusters to obtain information on the magnetic fields in galaxy clusters (Böhringer et al. 2016).

One of the most exciting applications of this sample is the study of the large scale matter distribution of the nearby Universe out to redshifts up to $z \sim 0.3$. In Böhringer et al. (2015) we have already studied the mass distribution in the nearby Universe in the southern sky. With the completion of the all-sky survey we can study the very interesting aspect of the dipole in the density distribution of the Universe that gives rise to the local bulk flow. Having reached a homogenisation of the ROSAT cluster sample across the sky, this data set is now perfectly suited for this study.

We like to thank the ROSAT team at MPE for the support with the data of the RASS and the staff at the German-Spanish Calar Alto observatory for the technical support during the observing runs. H.B. and G.C. acknowledge support from the DFG Transregio Program TR33 and the Munich Excellence Cluster "Structure and Evolution of the Universe". G.C. acknowledges support from Deutsches Zentrum für Luft- und Raumfahrt under grant 50OR1601. This research made use of the NASA/IPAC Extragalactic Database (NED), which is operated by the Jet Propulsion Laboratory under contract by NASA. We like to thank the anonymous referee for helpful comments.

\section{APPENDIX}

\section{A. EFFECT OF SAMPLE INCOMPLETENESS}

In section 8 we discussed the effect of sample incompleteness on the derived cosmological parameter constraints. In these studies we have removed clusters from or added clusters to the sample in a statistically random fashion. In reality we expect the last $3 \%$ of the clusters left without redshift not to have average properties, but rather to be for example mostly close to the flux limit, not very nearby, not the easiest to recognise in optical images. Therefore it is interesting to explore the incompleteness effect on the cosmological constraints when the missing clusters have preferentially low or high X-ray luminosity. To this end we statistically removed $3 \%$ of the clusters in the luminosity range $L_{X}=0.25-1 \times 10^{44} \mathrm{erg} \mathrm{s}^{-1}$ for sample C. Note that clusters with $L_{X}<0.25 \times 10^{44} \mathrm{erg} \mathrm{s}^{-1}$ are not used for the comsmological parameter constraints, because the $L_{X}-M$ relation is not well calibrated in this range. For sample D we removed $3 \%$ of the clusters in the luminosity range $L_{X} \geq 3 \times 10^{44} \mathrm{erg} \mathrm{s}^{-1}$.

The results are shown in Fig. A1. The best fitting parameters move in opposite directions in the $\Omega_{m}-\sigma_{8}$ parameter plane. This can be understood from an inspection of Fig. B.1 in Böhringer et al. (2014). Removing high luminosity clusters makes the luminosity function steeper, which is better fit by a lower $\sigma_{8}$, while $\Omega_{m}$ will be increased to compensate for the lowering of the normalisation from a decrease of $\sigma_{8}$. The opposite effect is observed by removing low luminosity clusters which is flattening the luminosity function. 


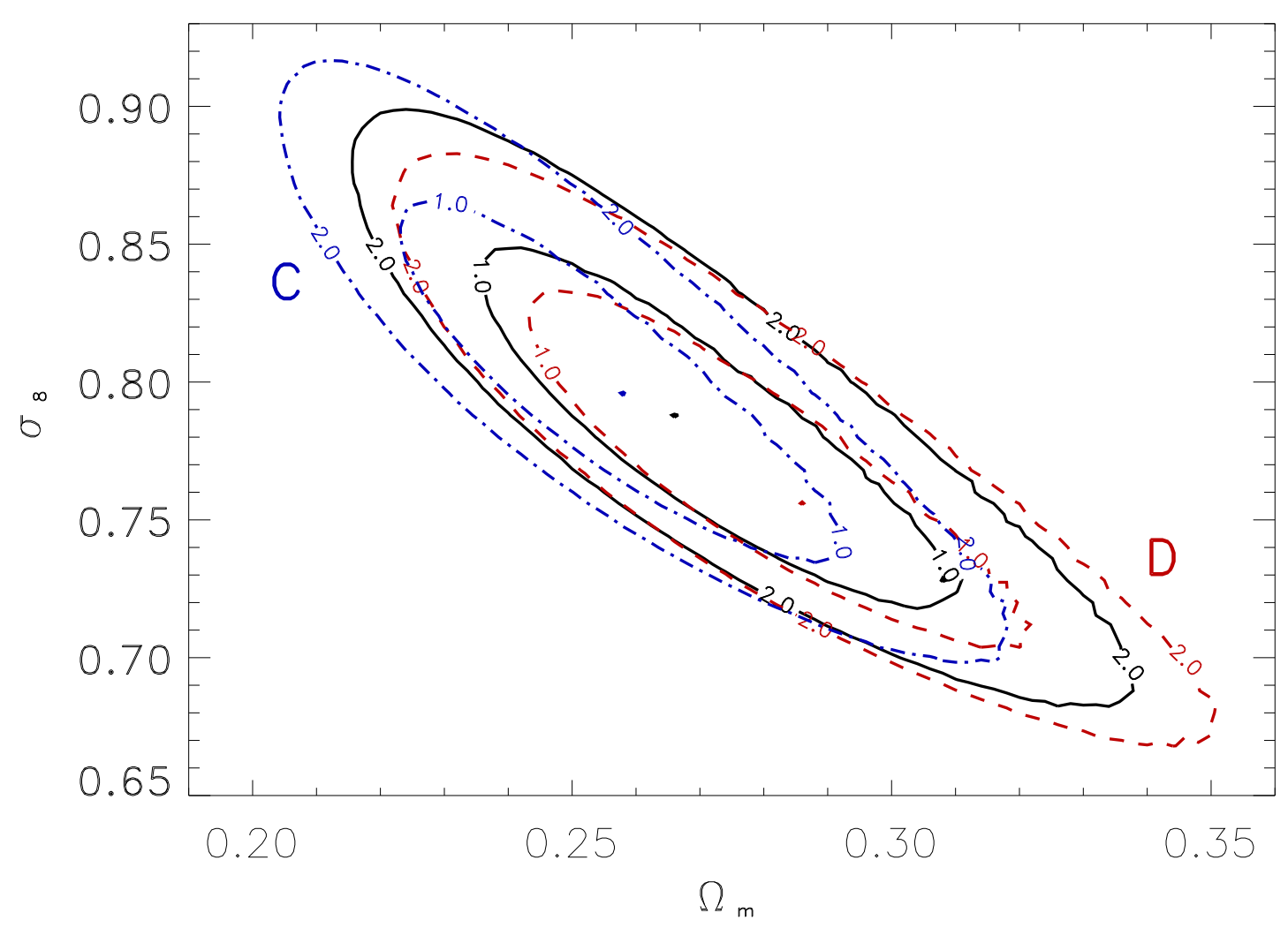

Figure AA1: Effect of an incomplete X-ray cluster sample on the derived cosmological parameter constraints. For sample $\mathrm{C}$ we have removed $3 \%$ low luminosity clusters, while sample D is depleted by clusters at high X-ray luminosity.

Table B2:. Sensitivity map of the NORAS II Survey.

\begin{tabular}{cccccc}
\hline \hline RA & DEC & S & size & g-long & g-lat \\
$(1)$ & $(2)$ & $(3)$ & $(4)$ & $(5)$ & $(6)$ \\
\hline 0.5000 & 89.5000 & 54.217 & 0.008726 & 122.8131 & 26.6396 \\
1.5000 & 89.5000 & 55.215 & 0.008726 & 122.8226 & 26.6378 \\
2.5000 & 89.5000 & 55.215 & 0.008726 & 122.8322 & 26.6362 \\
3.5000 & 89.5000 & 55.215 & 0.008726 & 122.8418 & 26.6347 \\
4.5000 & 89.5000 & 55.046 & 0.008726 & 122.8515 & 26.6334 \\
\hline
\end{tabular}

Note - Columns (1) and (2) provide the equatorial coordinates of the sky pixel for J2000 and columns (5) and (6) the corresponding galactic coordinates. Column (3) gives the number of photons that can be detected in that sky pixel for a source at the nominal flux limit. Column (4) gives the size of the sky pixel in square degrees. We show the first five lines of the Table, the full table is provided with the on-line data.

\section{B. SURVEY SENSITIVITY AND SELECTION FUNCTION}

In this section we provide the numerical data associated to Figs. 5 and 6 and the data of the survey mask. Table B2 provides the information given in Fig. 5, the equatorial and galactic coordinates of the sky pixels for the epoch J2000 and the number of photons that can be detected in the RASS at that pixel for a source at the limiting flux, $F_{X}=1.8 \times 10^{-12} \mathrm{erg} \mathrm{s}^{-1} \mathrm{~cm}^{-2}$.

Table B3 provides the data on the sky coverage as a function of flux shown in Fig. 6. It gives the fraction of the sky covered for a flux limit as quoted in the first column, based on a detection of at least 20 photons. 
Table B3:. Survey mask for NORAS II.

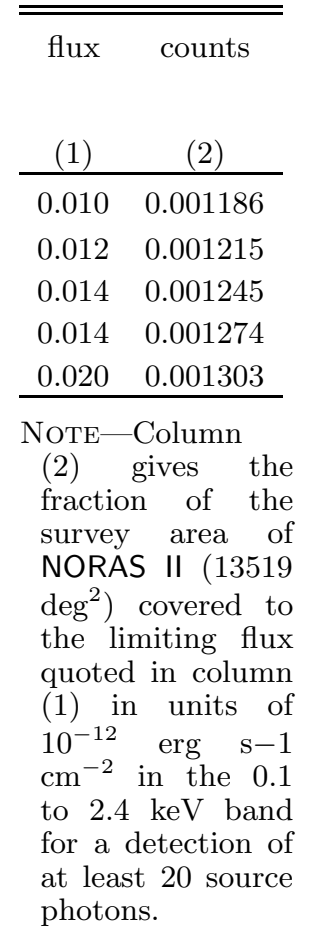

Table B4:. Survey mask for NORAS II.

\begin{tabular}{cccccc}
\hline \hline RA & DEC & $\mathrm{z}=0.005$ & $\mathrm{z}=0.01$ & $\ldots$ & $\mathrm{z}=0.8$ \\
$(1)$ & $(2)$ & $(3)$ & $(4)$ & $(5)$ & $(6)$ \\
\hline 0.5000 & 89.5000 & 0.00873 & 0.00145 & $\ldots$ & 38.90970 \\
1.5000 & 89.5000 & 0.00873 & 0.00145 & $\ldots$ & 38.90970 \\
\hline NoTE-The Table gives for every sky position with RA and \\
DEC given in columns (1) and (2) the minimim X-ray \\
luminosity (0.1 - 2.4 keV) to be detected with 20 source \\
photons at the redshifts given in the header. Here we give \\
the results only for 3 redshifts and show only the first two \\
lines of the Table. The full Table, which is provided with \\
the on-line data, lists the minimum luminosities for 160 \\
redshifts between $\mathrm{z}=0.005$ and 0.8.
\end{tabular}

Table B4 provides the the numerical values of the survey mask. It lists for each sky pixel the minimum X-ray luminosity required for a detection in the RASS with 20 source photons for 160 redshifts from $z=0.005$ to $z=0.8$.

\section{REFERENCES}

Anders, E. \& Grevesse, N., 1989, Geochim. Cosmochim. Acta, 53, 197

Arévalo, P., Churazov, E., Zhuravleva, I., et al., 2016, ApJ, 818, 14

Arnaud, M., Pratt, G.W., Piffaretti, R., et al., 2010, A\&A, 517, 92

Balaguera-Antolinez, A., Sanchez, A., Böhringer, H., et al., 2011, MNRAS, 413, 386

Balaguera-Antolinez, A., Sanchez, A., Böhringer, H., et al., 2012, MNRAS, 425, 2244

Bleem, L.E., Stadler, B., de Haan, T., et al., 2015, ApJS, 216, 27

Böhringer, H., Voges, W., Huchra, J.P., et al., 2000, ApJS, 129, 435
Böhringer, H., Schuecker, P., Guzzo, L., et al., 2001, A\&A, 369, 826

Böhringer, H., Collins, C.A., Guzzo, L., et al., 2002, ApJ, 566, 93

Böhringer, H., Schuecker, P., Guzzo, L., et al., 2004, A\&A, 425, 367

Böhringer, H., Schuecker, P., Pratt, G.W., et al., 2007, A\&A, 469, 363

Böhringer, H., Pratt, G.W., Arnaud, M., et al., 2010, A\&A, 514, 32

Böhringer, H., 2011, AIP Conf. Proceedings, 1381, 137

Böhringer, H., Dolag, K., Chon, G., 2012, A\&A, 539, 120 
Böhringer, H., Chon, G., Collins, C.A., et al., 2013, A\&A, 555, A30

Böhringer, H., Chon, G., Collins, C.A., et al., 2014, A\&A, 570, A31

Böhringer, H., Chon, M. Bristow, G., Collins, 2015, A\&A, 574, A26

Böhringer, H. \& Chon, 2015, A\&A, 574, L8

Böhringer, H. \& Chon, 2016, Mod. Phys. Let. A, 31, 1640008

Böhringer, H., Chon, G., Kronberg, P.P., 2016, A\&A, 596, A22

Borgani, S. Rosati, P., Tozzi, P., et al., 2001, ApJ, 561, 13

Bykov, A.M., Churazov, E.M., Ferrari, C., et al., 2015, SSRv, 188, 141

Cavaliere, A. \& Fusco-Femiano, R., 1976, A\&A, 49, 137

Chon, G., \& Böhringer, H., 2012, A\&A, 538, 35

Chon, G., \& Böhringer, H., 2013, MNRAS, 429, 3272

Chon, G., Bhringer, H., Zaroubi, S., 2015, A\&A, 575, L14

Churazov, E., Arevalo, P., Forman, W., et al. 2016, MNRAS, 463, 1057

Collins, C.A., Burke, D.J., Romer, A.K., et al. 1997, ApJ, 479, L117

Collins, C.A., Guzzo, L., Böhringer, H., et al., 2000, MNRAS, 319, 939

Croston, J.H., Pratt, G.W., Böhringer, H., et al., 2008, A\&A, 487,431

Cruddace, R., Voges, W., Böhringer, H., et al., 2002, ApJS, 140, 239

de Grandi, S., Böhringer, H., Guzzo, L., et al., 1999, ApJ, 514, 148

de Haan, T., Benson, B.A., Bleem, L.E., et al., 2016, arXiv1603.03904

Dickey, J.M. \& Lockman, F.J., 1990, ARA\&A, 28, 215

Ebeling, H., Voges, W., Böhringer, H., et al., 1996, MNRAS, 281, 799

Ebeling, H., Edge, A.C., Fabian, A.C., et al., 1997, ApJ, 479, 101

Ebeling, H., Edge, A.C., Bohringer, H., et al., 1998, MNRAS, 301,881

Ebeling, H., Edge, A.C., Henry, J. P. 2001, ApJ, 553, 668

Ebeling, H., Mullis, C.R., Tully, R.B., 2002, ApJ, 580, 774

Eckert, D., Roncarelli, M., Ettori, S., et al., 2015, MNRAS, 447, 2198

Einasto, J., Gramann, M., Saar, E., et al., 1993, MNRAS, 260, 705

Eisenstein D.J. \& Hu, W., 1998, ApJ, 458, 137

Guzzo, L., Schuecker, P., Böhringer, H.,et al., 2009, 499, 357

Hasselfield, M., Hilton, M., Marriage, T.A., et al., 2013, JACP, 7, 8

Henry, J.P., 2004, ApJ, 609, 603
Henry, J.P., Evrard, A.E., Hoekstra, H., et al., 2009, ApJ, 691, 1307

Kalberla, P.M.W., Burton, W.B., Hartmann, D., et al., 2005, A\&A, 440, 775

Kerscher, M., Mecke, K., Schuecker, P., et al., 2001, A\&A, 377, 1

Komatsu, E., Smith, K.M., Dunkley, J., et al., 2011, ApJS, 192, 18

Kravtsov, A.V. \& Borgani, S., 2012, ARA\&A, 50, 353

Lewis, A., Challinor, A., Lasenby, A., 2000, ApJ, 538, 473

Markevitch, M. \& Vikhlinin, A., 2007, PhR, 443, 1

Motl, P.M., Hallman, E.J., Burns, J.O., et al., 2005, ApJ, 623, L63

Mulchaey, J.S., Dressler, A., Oemler, A. (eds.), 2004, Clusters of Galaxies, Cambridge University Press

Ortiz-Gil, A., Guzzo, L., Schuecker, P., et al., 2004, MNRAS, 348, 325

Peacock, J.A. \& West, M., 1992, MNRAS, 259, 494

Perenod, S.C., 1980, ApJ, 236, 373

PLANCK Collaboration, Ade, P.A.R., Aghanim, N., et al.,

Planck 2013 results XVI, 2914, A\&A, 571, A20

Planck Collaboration 2015 results XXIV, 2016, A\&A, 294, A24

Planck Collaboration 2015 results XXVII, 2016, A\&A, 294, A27

Pratt, G.W., Croston, J. H.; Arnaud, M., et al., 2009, A\&A, 498, 361

Pratt, G.W., Arnaud, M., Piffaretti, R., et al., 2010, A\&A, 511A, 85

Reichardt, C.L., Stadler, B., Bleem, C.E., et al., 2013, ApJ, 763, 127

Reichert, A., Böhringer, H., Fassbender, R., Mhlegger, M., 2011, A\&A, 535, A4

Reiprich T.H. \& Böhringer, H., 2002, ApJ, 567, 716

Rozo, E., Wechsler, R.A., Rykoff, E.S., et al., 2010, ApJ, 708, 645

Rozo, E. \& Rykoff, E.S., 2014, ApJ, 783, 80

Schuecker, P., Böhringer, H., Guzzo, L., et al., 2001, A\&A,368, 86

Schuecker, P., Böhringer, H., Collins, C.A. et al., 2003a, A\&A, 398,867

Schuecker, P., Caldwell, R.R., Böhringer, H., et al., 2003b, A\&A, 402, 53

Tinker, J., Kravtsov, A.V., Klypin, a., et al., 2008, ApJ, 688, 709

Trümper, J., 1993, Science, 260, 1769

Vikhlinin, A., Kravtsov, A.V., Burenin, R.A., et al., 2009, ApJ, 692,1060

Voit, M., 2005, Rev. Mod. Phys., 77, 207

Voges, W., Aschenbach, B., Boller, T., et al. 1999, A\&A, 349, 389 